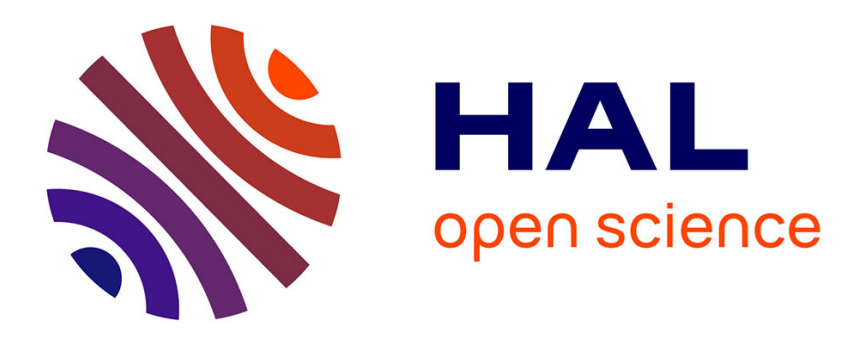

\title{
Effet Mössbauer et techniques complémentaires - II. - L'annihilation de positons \\ Chr. Janot
}

\section{To cite this version:}

Chr. Janot. Effet Mössbauer et techniques complémentaires - II. - L'annihilation de positons. Revue de Physique Appliquée, 1980, 15 (6), pp.1073-1077. 10.1051/rphysap:019800015060107300 . jpa00244825

\section{HAL Id: jpa-00244825 https://hal.science/jpa-00244825}

Submitted on 1 Jan 1980

HAL is a multi-disciplinary open access archive for the deposit and dissemination of scientific research documents, whether they are published or not. The documents may come from teaching and research institutions in France or abroad, or from public or private research centers.
L'archive ouverte pluridisciplinaire HAL, est destinée au dépôt et à la diffusion de documents scientifiques de niveau recherche, publiés ou non, émanant des établissements d'enseignement et de recherche français ou étrangers, des laboratoires publics ou privés. 


\title{
Effet Mössbauer et techniques complémentaires
}

\section{II. - L'annihilation de positons}

\author{
Chr. Janot \\ Laboratoire de Physique des Solides, Faculté des Sciences, C.O. n 140, 54037 Nancy Cedex, France
}

(Reçu le 14 novembre 1979, révisé le 28 janvier 1980, accepté le 29 janvier 1980)

\begin{abstract}
Résumé. - Les principes généraux des expériences utilisant l'annihilation de positons sont décrits. Des exemples de complémentarité avec l'effet Mössbauer sont donnés : mesure de densité électronique et de densité de spin, étude des défauts de structure et des mécanismes de diffusion, caractérisation des matériaux amorphes.
\end{abstract}

\begin{abstract}
General features of positron annihilation experiments are reported. Typical investigations in physics using positron and Mössbauer techniques are proposed : measurement of charge and spin density, study of structural defects and atomic transport mechanism, characterization of amorphous materials.
\end{abstract}

1. Principes généraux de la méthode. - Le positon est l'antiparticule de l'électron dont, par conséquent, il possède la masse $\left(m_{0} c^{2}=0,511 \mathrm{MeV}\right)$; sa charge est égale à $+\mathrm{e}$ et il possède un spin $1 / 2$, avec un moment magnétique parallèle.

Si un positon pénètre dans la matière, il est rapidement thermalisé par collisions inélastiques et son énergie décroît jusqu'à une valeur de l'ordre de $k_{\mathrm{B}} t$. Le temps de thermalisation dépend du type de matériau; il est de l'ordre de $10^{-12} \mathrm{~s}$ dans les solides. Après quoi, le positon s'annihile avec un électron assez éloigné des ions positifs qui repoussent les positons par interactions coulombiennes; ainsi, on peut prévoir que l'annihilation se fera préférentiellement avec les électrons de conduction dans les métaux (de préférence au voisinage de zones lacunaires ou d'impuretés de plus faible valence) avec des électrons extérieurs des ions négatifs dans les cristaux ioniques, et près des régions de faible densité dans les solides moléculaires.

Les paramètres d'annihilation sont sensibles à la structure électronique (densité et symétries) et fortement influencés par la présence d'imperfections dans le cas de solides cristallisés. Plusieurs articles de synthèses ont été publiés $[1,2,3,4,5,6,7$ et 8$]$; outre une information scientifique détaillée, ils contiennent un nombre impressionnant de références à des articles originaux que nous ne citerons pas ici en détail.

1.1 Processus D'ANNIHILATION ET PaRamètres CARACTÉRISTIQUES. - a) Une paire positon-électron peut s'annihiler suivant trois types de processus :
- annihilation libre,

- formation intermédiaire de positonium,

- existence d'un état piégé.

Dans le processus d'annihilation libre, les photons émis ont une énergie de l'ordre de $m_{0} c^{2}=0,511 \mathrm{MeV}$ bien supérieure à l'énergie initiale de l'électron et du positon. Le centre de masse du système est donc pratiquement au repos, ce qui implique le plus souvent l'émission de deux photons dans des directions sensiblement opposées (processus dit à $2 \gamma$ ).

Le positon peut occasionnellement former un état lié avec un électron juste avant l'annihilation. On obtient alors un atome dit positonium $\mathrm{P}_{\mathrm{S}}$, analogue à l'atome d'hydrogène. Pour que l'atome $P_{S}$ se forme, certaines conditions doivent être réalisées quant à l'énergie du positon et les états de l'électron. Ces conditions peuvent parfois être obtenues dans les gaz et les cristaux moléculaires. Cela ne se produit jamais dans les cristaux ioniques ou les métaux, sauf au voisinage des surfaces.

Le troisième processus, dit annihilation en états piégés, apparaît lorsque le matériau qui accueille les positons est électriquement inhomogène (ions négatifs, lacunes d'ions positifs, etc.). L'annihilation se produit alors préférentiellement près des sites déficitaires en charges positives.

Citons enfin une dernière possibilité qui vient d'être observée [9] où l'annihilation ne se produit pas dans le matériau étudié. Les positons injectés dans le cristal sont réémis à l'extérieur en faisceaux mono- 
chromatiques diffractés; il s'agit là d'une méthode potentielle très fine pour étudier la structure des surfaces ou des films minces (amorphes en particulier).

b) En se limitant au processus d'annihilation à 2 photons $\gamma$, en état libre ou piégé, les paramètres caractéristiques qui peuvent être mesurés sont

- le temps de vie du positon,

- la distribution angulaire et énergétique des photons d'annihilation.

S'il n'y a qu'un seul mécanisme d'annihilation, le nombre de positons décroît suivant une loi du type :

soit

$$
\mathrm{d} N=-\lambda N \mathrm{~d} t
$$

$$
N(t)=N_{0} \mathrm{e}^{-\lambda t} .
$$

Le graphe $\log N(t)$ est alors une droite de pente $\lambda$, inverse du temps de vie $\tau$ des positons. La mesure de $N(t)$, ou plus exactement du nombre d'annihilations à chaque instant se fait par une détermination du nombre des coïncidences retardées (en fonction du temps de retard) en tre l'émission d'un photon d'annihilation et celle d'un autre photon $\gamma$ émis en cascade avec le $\beta^{+}$. Ceci implique évidemment un choix judicieux des sources de positons $\left({ }^{22} \mathrm{Na},{ }^{58} \mathrm{Co}\right)$. Lorsque plusieurs processus d'annihilations sont superposés, on a :

$$
\frac{\mathrm{d} N}{\mathrm{~d} T}=-N_{\mathrm{o}} \sum_{\mathrm{i}} a_{\mathrm{i}} \lambda_{\mathrm{i}} \mathrm{e}^{-\lambda_{\mathrm{i}} t}
$$

et la détermination des temps de vie $\tau_{i}=\lambda_{i}^{-1}$ avec les contributions relatives $a_{\mathrm{i}}$ des différents mécanismes résulte d'une déconvolution des courbes expérimentales $N(t)$. Les $\tau_{\mathrm{i}}$ mesurés sont de l'ordre de $10^{-10} \mathrm{~s}$ et peuvent atteindre $10^{-7} \mathrm{~s}$ s'il y a formation de positonium.

Si le positon et l'électron s'annihilaient au repos, les deux photons $\gamma$ seraient émis strictement à $180^{\circ}$ l'un de l'autre et emporteraient chacun une énergie égale à $m_{0} c^{2}=0,511 \mathrm{eV}$. Si le positon thermalisé peut bien être considéré au repos, l'électron possède une quantité de mouvement et une énergie. La conservation de la quantité de mouvement totale est schématisée à la figure 1 . On voit donc qu'un électron de quantité de mouvement $\mathbf{p}_{\text {- entraîne une annihilation }}$ telle que les deux photons $\gamma$ sont émis à $\pi-\theta$ l'un de l'autre avec :

$$
\begin{aligned}
& p_{y} \simeq m_{0} c \frac{\theta^{2}}{2} \\
& p_{z} \simeq m_{0} c \theta
\end{aligned}
$$

où $p_{y}$ et $p_{z}$ sont les projections de $\mathbf{p}_{-}$parallèlement et perpendiculairement à la direction de propagation des $\gamma$ d'annihilation.

De même l'énergie de ces photons sera

$$
E_{i}=m_{0} c^{2}+\frac{1}{2} E_{-}
$$

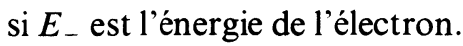

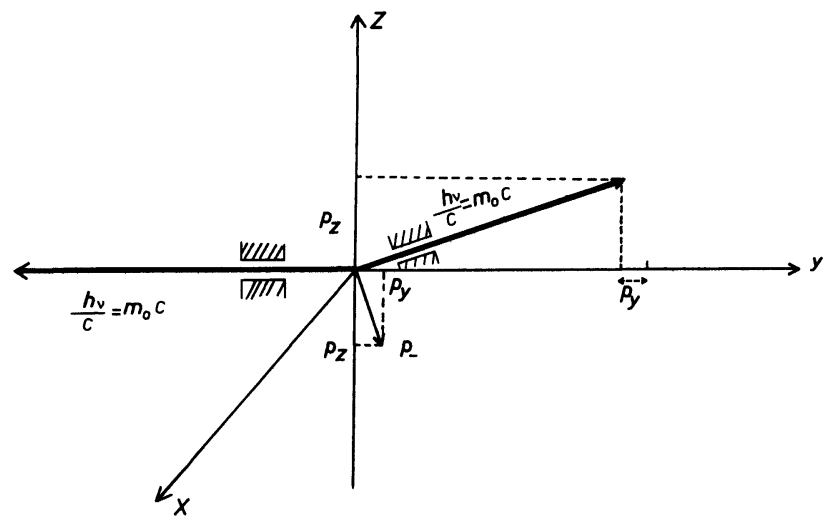

Fig. 1. - Représentation schématique de la conservation de la quantité de mouvement dans l'annihilation d'une paire $\mathrm{e}^{-} \cdot \mathrm{e}^{+}$.

[Pictural presentation of the momentum conservation in annihilarion of one electron-positron pair.]

La technique dite de corrélation angulaire permet de mesurer un taux d'annihilation $N(\theta)$ en fonction de $\theta$, en détectant simultanément les 2 photons d'annihilation en coïncidence. On limite ici l'exploration aux annihilations mettant en jeu des électrons se déplaçant dans un plan, en sélectionnant les $\gamma$ par des fentes verticales. Il est possible, mais plus délicat, de sélectionner les photons $\gamma$ par des trous et d'explorer l'espace des moments par des comptages de coïncidences $N(\theta, \psi)$ dans toutes les directions de l'espace autour de $O y$. Ces techniques sont assez lourdes du point de vue expérimental; la source est généralement du ${ }^{64} \mathrm{Cu}$ (plusieurs Curies); le domaine angulaire exploré est de quelques milliradians (6 à 20) avec une précision de 0,1 milliradian. La technique dite d'effet Döppler consiste à mesurer la distribution en énergie $N\left(E_{\gamma}\right)$ par une analyse d'amplitude du signal transmis par un détecteur solide, type $\mathrm{Ge}(\mathrm{Li})$; ce procédé beaucoup plus simple conduit à une résolution environ 10 fois moins bonne que la corrélation angulaire.

1.2 TyPeS D'INFORMATIONS DÉDUITES DES EXPÉRIENCES D'ANNIHILATION DE POSITONS. - Les mesures de temps de vie conduisent à une évaluation globale de la densité électronique $n_{-}$au point d'annihilation; on a en première approximation :

$$
\tau^{-1}=\lambda=\pi r_{0}^{2} c n_{-}
$$

$\left(r_{0}=\frac{e^{2}}{m_{0} c^{2}}\right.$ rayon de l'électron, $c$ célérité de la lumière dans le vide).

Les mesures de corrélation angulaire et d'effet Döppler donnent directement les distributions en moment $N\left(\mathbf{p}_{-}\right)$et en énergie $N\left(E_{-}\right)$de ces mêmes électrons. Il est immédiat de montrer que si l'annihilation a lieu uniquement avec des électrons de conduction libres, la courbe de corrélation angulaire est une parabole inversée s'annulant pour $\theta_{\mathrm{F}}=\hbar k_{\mathrm{F}} / m_{0} c$; on a alors une mesure directe du rayon $k_{\mathrm{F}}$ de la sphère de Fermi (méthode intéressante pour les amorphes 
par exemple). La présence d'une composante plus large dans $N(\theta)$, pour $\theta>\theta_{\mathrm{F}}$ (voir Fig. 2) indique une annihilation avec des électrons non libres (cœur, $3 \mathrm{~d}, \ldots)$. Le tracé complet des corrélations $N(\theta, \psi)$ conduit à la description de la surface de Fermi du matériau ; la technique est intéressante pour l'étude des alliages ou les corps mal ou peu cristallisés pour lesquels les moyens précis usuels (effet Haas-Van Halphen) sont inutilisables.

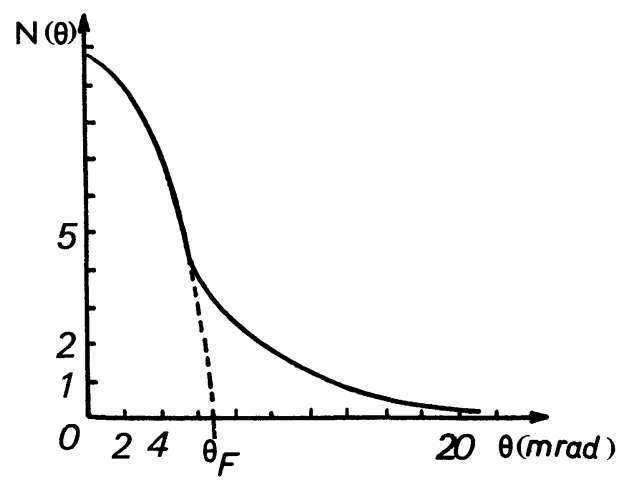

Fig. 2. - Courbe typique de distribution angulaire des photons d'annihilation dans un métal.

[Typical angular correlation curve in a metal.]

Les courbes de corrélation angulaire peuvent, dans le cas de matériaux magnétiques, donner une sorte de mesure de la polarisation de spin en même temps qu'une confirmation que la contribution de cœur vient surtout des électrons d.

Les positons émis durant la désintégration $\beta^{+}$ de la source sont partiellement polarisés dans le sens de leur mouvement; cette polarisation est conservée jusqu'à l'annihilation.

Si on applique un champ magnétique extérieur à un matériau ferromagnétique, il y a orientation préférentielle des spins des électrons non appariés (par exemple les électrons $3 \mathrm{~d}$ dans les métaux de la première série de transitions). Soit $N(\theta) \uparrow \uparrow$ et $N(\theta) \uparrow \downarrow$ les courbes de corrélations angulaires obtenues suivant que les spins électroniques sont orientés par le champ appliqué parallèlement ou antiparallèlement au faisceau de positons. On doit avoir :

$$
\begin{aligned}
& N(\theta) \downarrow \uparrow>N(\theta) \\
& N(\theta) \uparrow \uparrow<N(\theta)
\end{aligned}
$$

puisqu'il y a respectivement augmentation et diminution des interactions singulets entre positons et électrons $3 d$. Le rapport

$$
P(\theta)=\frac{N(\theta) \uparrow \downarrow-N(\theta) \uparrow \uparrow}{N(\theta) \uparrow \downarrow+N(\theta) \uparrow \uparrow}
$$

donne pour chaque valeur de $\theta$, donc de $P_{z}$, une mesure de la densité de spin. En fait, une véritable mesure demanderait, là encore, la connaissance des fonctions d'onde du positon et des électrons.
Le point intéressant est de $P(\theta)>0$ pour $\theta>\theta_{\mathrm{F}}$, c'est-à-dire pour la contribution de cœur, ce qui confirme la participation des électrons $3 \mathrm{~d}$ à cette contribution de cœur (alliages de fer, nickel, zinc, cuivre) [10].

Dans certains cas, comme le fer monocristallin, on a obtenu $P(\theta)<0$ pour $0<\theta<\theta_{\mathrm{F}}$, ce qui semble indiquer une polarisation des électrons $4 \mathrm{~s}$ antiparallèlement aux électrons $3 \mathrm{~d}$.

Une autre application typique concerne l'étude des défauts de structure, entraînant une annihilation du positon sous forme piégée. Dans le cas de lacune dans un métal par exemple, il y a des sites où il manque des ions positifs et où par conséquent le positon ira s'annihiler de préférence. Comme la densité électronique est ici plus faible que pour les sites normaux du réseau, le temps de vie augmente, la composante parabolique de $N(\theta)$ voit sa contribution amplifiée et la distribution $N\left(E_{\gamma}\right)$ s'affine. On peut alors montrer par exemple [11] que le temps de vie moyenne $\bar{\tau}(T)$ évolue en fonction de la température suivant une courbe (Fig. 3) présentant un point d'inflexion à la

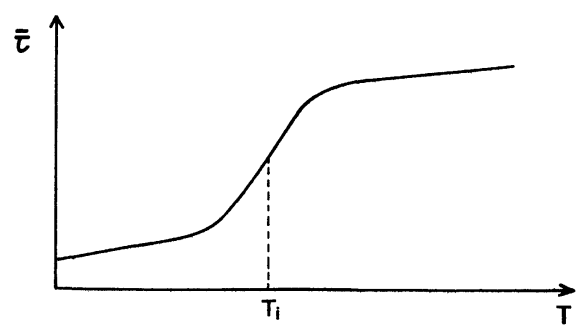

Fig. 3. - Courbe typique de l'évolution du temps de vie des positons dans un métal en fonction de la température.

[Typical temperature dependence of the positron life time in a metal.]

température $T_{\mathrm{i}}$; l'énergie de formation des lacunes $E_{\mathrm{F}}$ est alors donnée par :

$$
E_{\mathrm{F}} \simeq \frac{-2 k}{\bar{\tau}\left(T_{\mathrm{i}}\right)-\tau_{0}\left(T_{\mathrm{i}}\right)} \mathrm{d} \bar{\tau} / \mathrm{d}(1 / T)_{\mathrm{i}}
$$

( $\tau_{0}$ temps de vie dans le réseau sans lacunes extrapolé à la température $T_{\mathrm{i}}$ ). On peut atteindre de la même manière les impuretés, les associations de défauts, les dislocations, les précipités, etc. [7].

2. Quelques applications et complémentarité avec la spectrométrie Mössbauer. - 2.1 ANNIHILATION DE POSITONS ET DÉPLACEMENT ISOMÉRIQUE. - Le temps de vie mesure la densité totale d'électrons au point d'annihilation, c'est-à-dire en position interstitielle des atomes dans un réseau sans défauts. En corrélation angulaire les composantes étroite (parabolique) et large (gaussienne) mesurent la distribution en moments et la contribution des électrons libres (s ?) et des électrons de cour (d ?) respectivement. Par ailleurs, le déplacement isomérique est proportionnel à la densité électronique (de type s) au noyau. Dans 
un alliage, la variation de ces paramètres en fonction de la composition est influencée à la fois par l'effet de taille (variation du volume atomique) et les effets de transfert de charges éventuels (d'une bande de type $s$ vers une bande de type d par exemple). Considérons par exemple un alliage binaire du type $\mathrm{MT}_{x} \mathbf{M}_{1-x}$ où $\mathrm{MT}$ est un métal de transition ayant une bande $\mathrm{d}$ et une bande $\mathrm{s}$ incomplètes, $\mathrm{M}$ un métal normal ayant une forte densité d'électron s. Une mesure du temps de vie $\tau(x)$ en fonction de $x$ peut donner :

- une variation linéaire depuis $\tau_{0}$ pour $\mathbf{M}$ pur jusque $\tau_{1}$ pour MT pur; dans ce cas, on peut conclure que l'effet de volume est négligeable et que les atomes gardent un volume insensible à la composition dans l'alliage; les variations de déplacements isomériques et les mesures de corrélation angulaire peuvent alors être interprétées en terme de transfert de charges $\mathrm{s} \rightarrow \mathrm{d}$ uniquement,

- ou bien $\tau(x)$ ne varie pas linéairement avec $x$; la correction à la linéarité permet alors d'estimer l'effet de volume et de poursuivre ensuite l'interprétation comme précédemment [12].

On peut aussi corréler les résultats des expériences Mössbauer et d'annihilation de positons pour déterminer les densités de spin des électrons. Il s'agit alors de comparer les mesures de champ magnétique hyperfin avec le paramètre $P(\theta)$ de corrélation angulaire sous champ magnétique extérieur (voir section 1.2).

Peu d'expériences ont été réalisées dans ce sens malgré tout l'intérêt qu'elles présentent.

2.2 DéfaUts ET MÉCANISMES DE DIFfUSION. - Dans un milieu isotrope, le coefficient de diffusion atomique $D$ par un mécanisme de saut peut être obtenu dans certains cas par l'élargissement $\Delta \Gamma$ des pics Mössbauer. On a [13] :

$$
D\left(l^{2}\right)=\frac{\Delta \Gamma}{12 h} l^{2}
$$

où $l^{2}$ est la valeur quadratique moyenne du déplacement atomique global par échange avec un défaut (lacune, bilacune, ...). Si on mesure par ailleurs directement $D\left(l^{2}\right)$ par une méthode macroscopique, il est alors possible de calculer $l^{2}$ et d'en déduire le type de défauts dirigeant la diffusion.

Pour le système $V^{57} \mathrm{Co}^{*}$ par exemple [14] on trouve $l$ égal à une distance atomique $a_{0}$ pour $T \lesssim 1400^{\circ} \mathrm{C}$, ce qui correspond à un mécanisme classique monolacunaire pur. Au-dessus de $1500^{\circ} \mathrm{C}, l$ devient voisin de $1,3 a_{0}$, ce qui peut être interprété par une contribution faible mais mesurable de bilacunes [14]; on peut calculer [14] qu'un mécanisme bilacunaire pur dans un cubique centré comme le vanadium conduirait à $l=1,66 a_{0}$; dans un mécanisme mixte, monolacunes + bilacunes, on aura les relations suivantes :

$$
\begin{aligned}
\Delta \Gamma_{\text {total }} & =\Delta \Gamma_{1 \mathrm{v}}+\Delta \Gamma_{2 \mathrm{v}} \\
D_{\text {total }} & =D_{1 \mathrm{v}}+D_{2 \mathrm{v}}=\frac{\left(k a_{0}\right)^{2}}{12 h} \Delta \Gamma_{\text {total }} \\
D_{1 \mathrm{v}} & =\frac{a_{0}^{2}}{12 h} \Delta \Gamma_{1 \mathrm{v}} \\
D_{2 \mathrm{v}} & =\frac{\left(1,66 a_{0}\right)^{2}}{12 h} \Delta \Gamma_{2 \mathrm{v}} .
\end{aligned}
$$

De ces équations, on peut par exemple tirer le rapport $D_{2 \mathrm{v}} / D_{\mathrm{iv}}$, en fonction des mesures. Dans l'exemple précédent, on trouve $D_{2 \mathrm{v}} / D_{1 \mathrm{v}}=1,7$. Ce rapport s'exprime par ailleurs en fonction des concentrations relatives de mono- $C_{1 \mathrm{v}}$ et bilacunes $C_{2 \mathrm{v}}$ et de la différence $\Delta E_{\mathrm{m}}$ entre les énergies de migration de ces défauts, soit :

$$
\frac{D_{2 \mathrm{v}}}{D_{1 \mathrm{v}}} \simeq \frac{C_{2 \mathrm{v}}}{C_{1 \mathrm{v}}} \exp \frac{\Delta E_{\mathrm{m}}}{k T}=1,7
$$

avec

$$
\frac{C_{2 \mathrm{v}}}{C_{1 \mathrm{~V}}} \simeq \exp -\frac{E_{\mathrm{F}}^{1 \mathrm{v}}-E_{\mathrm{L}}}{k T} .
$$

On peut alors mesurer les énergies de formation $E_{\mathrm{F}}^{1 \mathrm{~V}}=2,2$ eV et $E_{\mathrm{F}}^{2 \mathrm{~V}}=2 E_{\mathrm{F}}^{1 \mathrm{v}}-E_{\mathrm{L}}$ des monoet bilacunes par la variation $\tau(T)$ du temps de vie des positons. Si on y ajoute l'énergie d'activation

$$
E_{\mathrm{a}}=E_{\mathrm{F}}^{1 \mathrm{v}}+E_{\mathrm{m}}^{1 \mathrm{v}}=3,4 \mathrm{eV}
$$

mesurée directement par une méthode macroscopique, tous les paramètres des défauts sont alors calculables. Ici par exemple on obtient :

$$
\begin{gathered}
E_{\mathrm{F}}^{1 \mathrm{v}}=2,2 \mathrm{eV} ; \quad E_{\mathrm{m}}^{1 \mathrm{v}}=1,2 \mathrm{eV} \\
E_{\mathrm{L}} \simeq 0,7 \mathrm{eV} ; \quad E_{\mathrm{F}}^{2 \mathrm{~V}}=3,7 \mathrm{eV} ; \quad E_{\mathrm{m}}^{2 \mathrm{~V}} \simeq 0,75 \mathrm{eV} \\
C_{2 \mathrm{~V}} / C_{1 \mathrm{v}} \simeq 0,15 \text { à } 1500^{\circ} \mathrm{C} .
\end{gathered}
$$

2.3 ETUDE DES MATÉRIAUX AMORPHES. - On verra par ailleurs [15] comment l'effet Mössbauer peut être utilement employé pour l'étude des propriétés, notamment magnétiques, des matériaux amorphes. D'intéressantes informations complémentaires peuvent être obtenues par des expériences d'annihilation de positons. On peut citer par exemple :

- la mise en évidence expérimentale, par mesure de temps de vie ou de corrélation angulaire, que la diffusion ou la déformation plastique se fait sans création de défauts (lacunes ou dislocations) pour les matériaux amorphes. Le temps de vie, ou la composante étroite de $N(\theta)$, reste en effet constant quelle que soit la température, ou le taux de déformation (obtenu par laminage) [16],

- la possibilité de raffiner la connaissance des propriétés magnétiques en déterminant notamment le signe de $P(\theta)$ (corrélation angulaire sous champ extérieur) suivant les valeurs de $\theta$, ce qui conduit à la nature de la polarisation de spin (s ou d) en fonction de la composition, 
- la mesure directe du rayon $k_{\mathrm{f}}$ de la sphère de Fermi qui est une donnée nécessaire à l'interprétation des mesures de résistivité dans les amorphes [17],
- l'évolution in situ de la compacité des matériaux amorphes (relaxation de structure, recuit, etc.).

\section{Bibliographie}

[1] West, R. N., Adv. Phys. 22 (1973) no 3.

2] Seeger, A., J. Phys. F 3 (1973) 248.

[3] Doyama, M., Hasiguti, R. R., Cryst. Lattice Defects 4 (1973) 139.

[4] Dekhtyar, I. YA, Physics Report (Phys. Lett. C) 9C (1974) 245.

[5] Goldanski, V. I., Revue d'Energie atomique (Ed. par I.A.E.A.) $6(1968)$.

[6] Stewart, A. T., Roellig, L. O., Positron annihilation (Academic Press, New York et London), 1967.

[7] Doyama, M., in Progress in the study of point defects (Ed. by Doyama and Yoshida S.), 1977, p. 3.

[8] Janot, C., Rev. Phys. Appl. 11 (1976) 89.

[9] Mills, A. P., Platzman, P. M. and Brown, B. L., Phys. Rev. Lett. 41 (1978) 1076.
[10] Mijnarends, P. E., Physica 63 (1973) 248.

[11] Janot, C., George, G. and Boldron, M., J. Physique 40 (1979) 39.

[12] Dezsi, I., Balogh, A., Balogh, J., KaJcsos, Zs. and NaJY, D. L., Proc. Int. Conf. Mössbauer spectroscopy, V. 1, p. 377, Bucarest (1977).

[13] Janot, C., J. Physique 37 (1976) 253.

[14] Janot, C. and Delcroix, P., J. Physique Colloq. 40 (1979) C2-650.

[15] Voir article sur amorphes dans ce volume.

[16] Chen, H. S. and Chuang, S. Y., J. Electron. Mater. 4 (1975) 783. Appl. Phys. Lett. 27 (1975) 316.

[17] Mangin, P. and Marchal, G., Phys. Lett. 68A (1978) 466. 